\title{
Present-Day Carbon Abundances of Early-Type Stars
}

\section{F. Nieva ${ }^{\star a b}$ and N. Przybilla ${ }^{a}$}

${ }^{a}$ Dr. Remeis-Sternwarte Bamberg, Sternwartstrasse 7, D-96049 Bamberg, Germany

${ }^{b}$ Observatório Nacional, Rua G. José Cristino 77, 20921-400, Rio de Janeiro, Brazil

E-mail: nieva@sternwarte.uni-erlangen.de

Carbon is one of the most abundant metals in the universe because of its synthesis in the fundamental $3 \alpha$ reaction. The knowledge of carbon abundances in different environments is one key ingredient to our understanding of stellar and galactochemical evolution. Studies of luminous OB-type stars allow us to address both topics even in galaxies beyond our own. Unfortunately the history of carbon abundance determinations from these objects in the last three decades is one of limited success. Analyses of the strong and weak line spectra of C II as well as C III tend to be largely discrepant. We present results of quantitative spectral analyses based on a sophisticated model atom for non-LTE line-formation calculations of C II-IV. As a first application, carbon abundances in a sample of B-type dwarfs and giants in nearby associations and in the field are determined. Consistency is finally achieved for all measurable lines from the three ionization stages. This includes in particular the notorious C II $\lambda \lambda 4267$ and $6578 / 6582 \AA$ features which are highly important for abundance determinations of fast-rotating and extragalactic objects. The long-standing problem of carbon line formation can now be regarded as solved, with the previous difficulties related to the use of inaccurate atomic data and stellar parameters. A highly homogeneous and slightly sub-solar present-day carbon abundance from young stars in the solar vicinity of $\log \mathrm{C} / \mathrm{H}+12=8.32 \pm 0.04$ is derived.

International Symposium on Nuclear Astrophysics - Nuclei in the Cosmos - IX

June 25-30 2006

CERN, Geneva, Switzerland

${ }^{*}$ Speaker. 


\section{Introduction}

One of the most abundant metals in the universe is carbon, produced in the $3 \alpha$ reaction in massive stars and constituting the central building block of all organic chemistry. Abundances derived from luminous OB stars provide important constrains on stellar and chemical evolution of our own and other galaxies. In extragalactic applications (e.g. observations of main sequence stars in the Magellanic Clouds, which have become feasible recently) astrophysicists desire to analyse the strongest features in the metal line spectra, as these are the only measurable at low signal-to-noise $(\mathrm{S} / \mathrm{N})$ and/or high projected rotational velocities. In the case of ionized carbon these are the prominent lines of the two multiplets C II $\lambda \lambda 4267.02 / 4267.27 \AA$ and $6578.03 / 6582.85 \AA$. These lines are unfortunately highly sensitive to non-LTE effects, as well as to the choice of stellar atmospheric parameters. So far, all studies from the literature failed to derive consistent abundances from these lines and also to establish the $\mathrm{C}$ II/III ionization equilibrium.

The present work aims to provide a solution to the classical non-LTE problem of carbon abundance determinations in OB stars. A reliable C II-IV model atom is developed and first applications on high-quality spectra are presented. Besides great care in the selection of atomic data, special emphasis is also given to an accurate atmospheric parameter determination, both in order to minimise systematic uncertainties. The entire measurable C II-IV spectrum in the visual is investigated.

\section{Model Calculations}

A hybrid approach is used for the non-LTE line-formation computations. These are based on line-blanketed plane-parallel, homogeneous and hydrostatic LTE model atmospheres calculated with ATLAS9. Non-LTE synthetic spectra are computed with recent versions of DETAIL and SURFACE. These codes solve the coupled radiative transfer and statistical equilibrium equations and compute synthetic spectra using refined line-broadening data, respectively. Non-LTE lineformation computations for $\mathrm{C}$ II/III/IV, hydrogen and $\mathrm{He} \mathrm{I} / \mathrm{II}$ are performed using state-of-the-art model atoms based on critically selected atomic data: [4], [6], [7] and [8].

\section{Observations and Analysis}

A first sample of six apparently slow-rotating Galactic B-type dwarfs and giants from OB associations and from the field in the solar vicinity is analysed. The observational database consists of very high S/N spectra obtained with FEROS on the $2.2 \mathrm{~m}$ telescope at La Silla, Chile (ESO).

The stellar parameters are derived from application of an extensive iterative method resulting in simultaneous fits to all measurable $\mathrm{H}, \mathrm{He}$ [5] and $\mathrm{C}$ II-IV lines [6]. The iteration is performed on effective temperature $T_{\text {eff }}$ and surface gravity $\log g$ (goal: to achieve ionization equilibrium) as well as the micro-, macroturbulent and projected rotational velocities (from carbon line profiles), $\mathrm{He}$ and $\mathrm{C}$ abundances and different sets of atomic data. The final solution for each star is obtained when the statistical deviations of the averaged $\mathrm{C}$ abundance achieve their minimum. By application of the procedure to all programme stars it was possible to calibrate the C II-IV model atom for the entire parameter range $\left(21500 \leq T_{\text {eff }} \leq 32000 \mathrm{~K}, 3.10 \leq \log g \leq 4.30\right.$, dwarfs and giants $)$ and simultaneously to obtain highly accurate $\mathrm{C}$ abundances for the sample. 


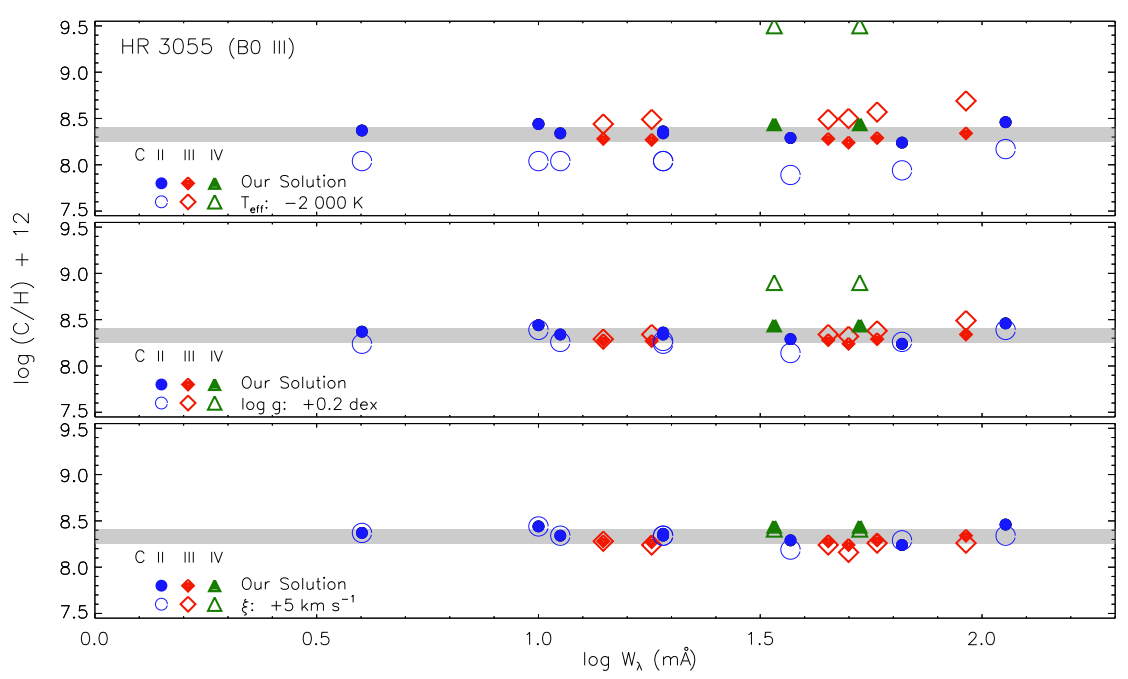

Figure 1: Sensitivity of carbon abundances to typical parameter variations found in the literature. Deviations from our solution for HR 3055 are shown for the 3 ionization stages. The grey rectangles correspond to $1 \sigma$ uncertainties of the stellar carbon abundance in our solution (upper left corner). The offsets of the parameters are also displayed.

\section{Sensitivity of Carbon Abundances to Parameter Variations}

Spectral lines of $\mathrm{C}$, as well as many other metal lines, can be highly sensitive to atmospheric parameter variations. This behaviour is utilised to find the best solution for both stellar parameters and abundances. In Fig. 1 non-LTE carbon abundances as a function of the equivalent width $W_{\lambda}$ in HR 3055 are displayed for our solution (see final parameters in Fig. 2) and variations in $T_{\text {eff }}, \log g$ and microturbulence. The offsets of the parameters are typical for systematic discrepancies found in the literature. Note that the C IV lines are extremely sensitive to changes in $T_{\text {eff }}$ and $\log g$ at this temperatures $(31200 \mathrm{~K}$ ), with discrepancies up to $+1.0 \mathrm{dex}$ in abundance. The $\mathrm{C}$ II/III ionization equilibrium is also never established for a variation of these parameters (abundance changes up to -0.40 dex for $\mathrm{C}$ II and up to +0.35 dex for $\mathrm{C}$ III compared to our solution). An expected reduction of the abundances from strong lines is obtained for an increased microturbulence. Note that the scale in carbon abundance used is large $(1.5 \mathrm{dex})$ and the variations of $\mathrm{C}$ abundances are significant relative to the high accuracy obtained in our solution. The systematic variation of the total carbon abundance can reach 0.1 dex and its statistical $1 \sigma$ value $\sim 0.4$ dex, when compared to our solution. These discrepancies increase when we use different sets of atomic input data.

\section{Results for the Programme Stars}

Non-LTE and LTE abundances vs. $W_{\lambda}$ for all individual lines are displayed in Fig. 2, showing excellent consistency in non-LTE. Identifications of the C II $\lambda \lambda 4267,6578 / 83,6151$ and $6462 \AA$ lines are displayed. These lines are highly sensitive to deviations from detailed equilibrium. The final parameters, as well as the carbon abundances, are also shown in Fig. 2. An immediate consequence of our careful analysis is a highly consistent value of carbon abundance free of systematic effects for all the stars of the sample. In Fig. 3 we show a comparison of our first results 


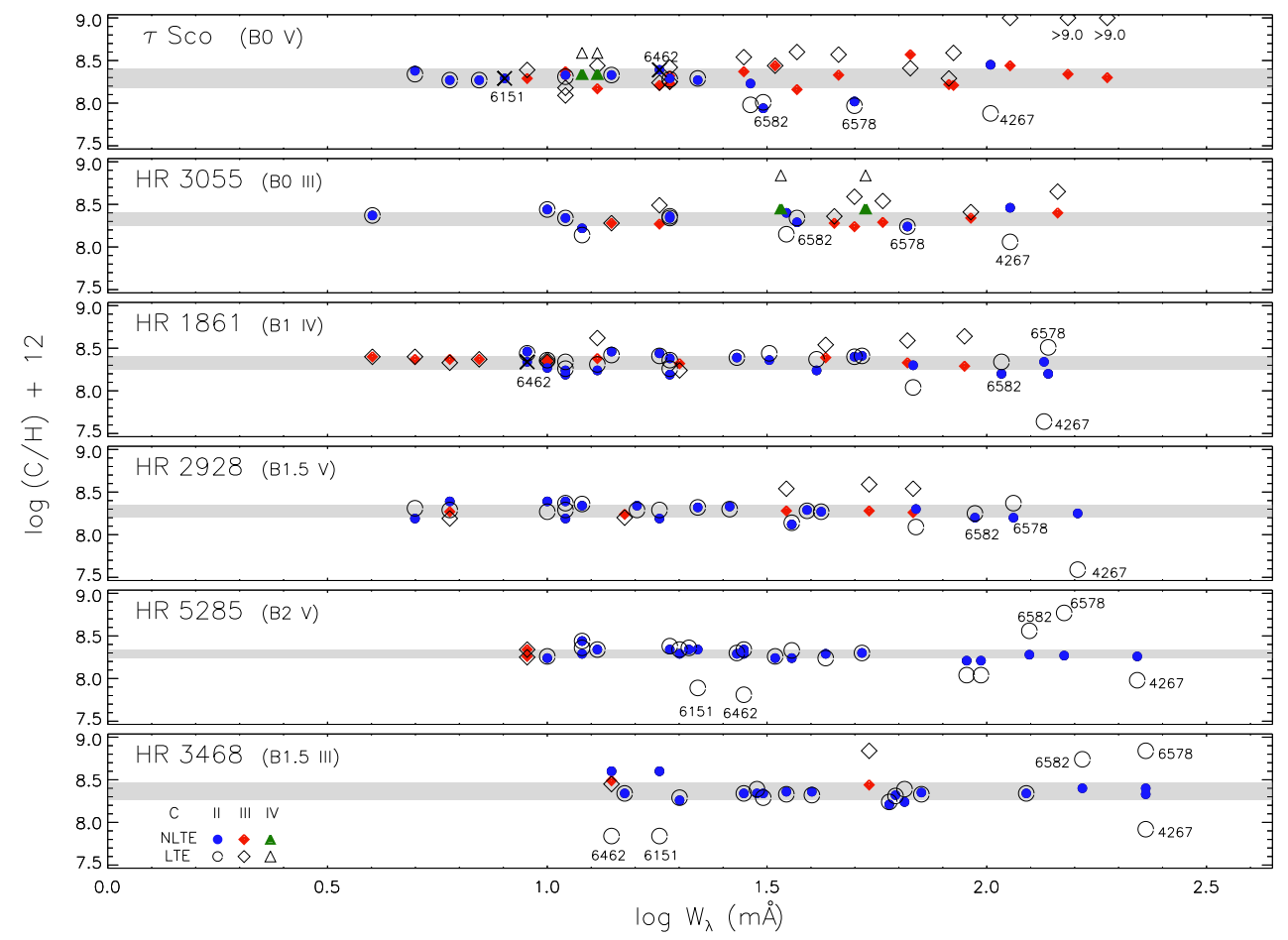

Figure 2: Non-LTE (filled symbols) and LTE (open symbols) abundances vs. equivalent width for C II-IV lines that could be measured in each spectrum. The ID of the stars and the derived mean carbon abundance is given in the upper left corner of each row, as well as derived basic stellar parameters $\left(T_{\text {eff }}, \log g\right.$, micro-, macroturbulent and projected rotational velocity). The grey rectangles correspond to $1 \sigma$-uncertainties of the stellar carbon abundance. Identification of lines with high sensitivity to non-LTE effects is displayed. Emission lines are marked by crosses (C II $\lambda \lambda 6151$ and $6462 \AA$ in $\tau$ Sco and $\lambda 6462 \AA$ in HR 1861): LTE calculations are not able to reproduce them even qualitatively.

for present-day carbon abundances of early B-type stars in the solar vicinity with results from the literature and with recent solar values (our stars coincide with 6 objects from [3]). Despite our - so far-small sample, it is clear that the fact of avoiding systematic effects in the whole analysis leads to a highly consistent value of carbon abundance. An analysis of a larger sample has to be done in order to improve on the statistics.

\section{Conclusions}

A highly consistent mean carbon abundance of $\varepsilon(C)=8.32 \pm 0.04$ is derived from the six sample stars, which provides a tight estimate to the present-day $\mathrm{C}$ abundance from young stars in the solar vicinity. The atmospheric composition appears to be unaffected by chemical mixing in the course of stellar evolution, i.e. we find no trend of $\mathrm{C}$ abundances with evolutionary age. For comparison, adopting results from [3] one derives a mean $\varepsilon(C)=8.19 \pm 0.12$ from the same six stars, implying a considerable systematic shift and a significantly increased statistical scatter. More objects need to be analysed in order to confirm such homogeneous present-day (slightly) sub-solar carbon abundances - considering as references $\varepsilon(\mathrm{C})_{\odot}=8.39 \pm 0.05$ [1] or $\varepsilon(\mathrm{C})_{\odot}=8.52 \pm 0.06$ [2] 


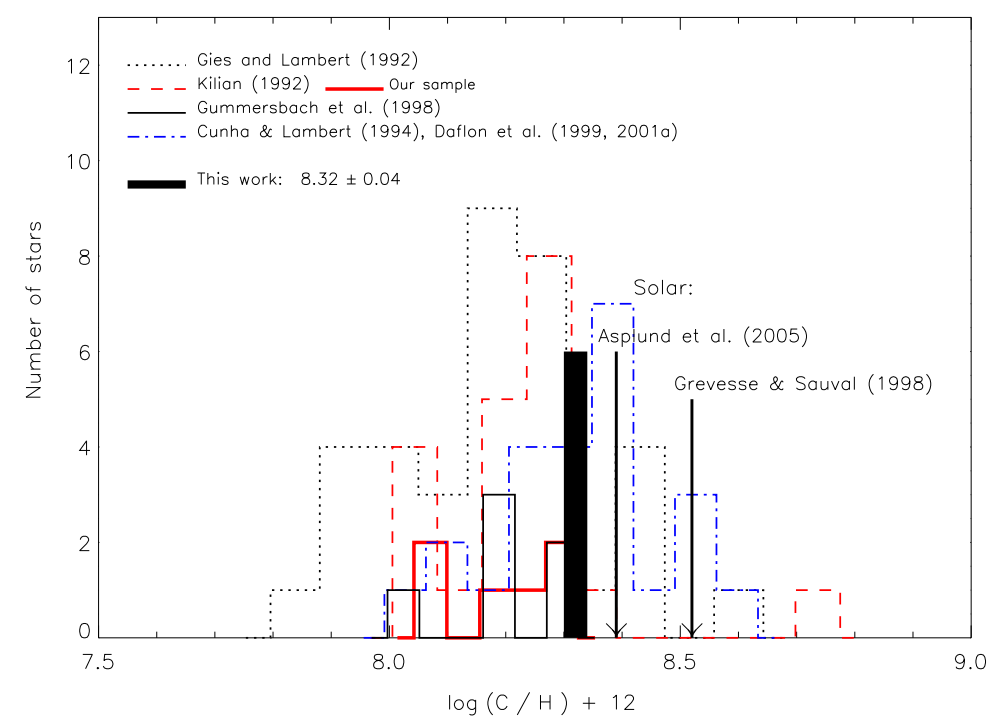

Figure 3: Comparison of carbon abundances derived from our sample of early B-type stars in the solar vicinity with results from the literature and with recent solar values.

- in nearby associations (HR 1861: Ori OB1; $\tau$ Sco, HR 5285: Sco-Cen) and in the field (the other stars). Despite the small sample size analysed so far, our highly accurate results indicate that the large scatter found for carbon abundances in early-type stars in previous work could be a consequence of systematic uncertainties introduced by the choice of inappropriate atomic data and stellar parameters.

\section{References}

[1] M. Asplund, N. Grevesse, A. J. Sauval, The Solar Chemical Composition, 2005, in Cosmic Abundances as Records of Stellar Evolution and Nucleosynthesis, ed. T. G. Barnes III \& F. N. Bash (San Francisco: ASP), 25

[2] N. Grevesse \& A. J. Sauval, Standard Solar Composition, 1998, Space Sci. Rev., 85, 161

[3] J. Kilian, Chemical abundances in early B-type stars, 1992, A\&A, 262, 171

[4] M. F. Nieva \& N. Przybilla, C II Abundances in Early-Type Stars: Solution to a Notorious Non-LTE Problem, 2006, ApJ, 639, L39

[5] M. F. Nieva \& N. Przybilla, Hydrogen and helium line-formation in OB dwarfs and giants. A hybrid non-LTE approach, 2007a, A\&A, in press (astro-ph/0608117)

[6] M. F. Nieva \& N. Przybilla, 2007b, submitted to A\&A

[7] N. Przybilla, Non-LTE modelling of the He I $10830 \AA$ Aine in early-type main sequence stars, 2005 , A\&A, 443, 293

[8] N. Przybilla, \& K. Butler, Non-LTE Line Formation for Hydrogen Revisited, 2004, ApJ, 609, 1181 